\title{
Narrativa
}

\section{Da Il bell'Antonio a Volevo i pantaloni. Patriarcato e Mascolinità}

Renato Ventura

\section{OpenEdition}

Edizione digitale

URL: https://journals.openedition.org/narrativa/537

DOI: 10.4000/narrativa.537

ISSN: 2804-1224

\section{Editore}

Presses universitaires de Paris Nanterre

\section{Edizione cartacea}

Data di pubblicazione: 1 décembre 2018

Paginazione: 81-92

ISBN: 978-2-84016-325-1

ISSN: $1166-3243$

\section{Notizia bibliografica digitale}

Renato Ventura, «Da I/ bell'Antonio a Volevo i pantaloni. Patriarcato e Mascolinità», Narrativa [Online], 40 | 2018, online dal 01 novembre 2021, consultato il 08 décembre 2021. URL: http://

journals.openedition.org/narrativa/537 ; DOI: https://doi.org/10.4000/narrativa.537

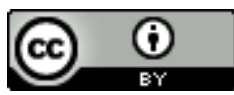

Narrativa est mise à disposition selon les termes de la Licence Creative Commons Attribution 4.0 International. 


\title{
Da Il bell'Antonio a Volevo i pantaloni. Patriarcato e Mascolinità
}

\begin{abstract}
RIASSUNTO
L'articolo analizza il legame tra patriarcato e mascolinità attraverso i romanzi di Vitaliano Brancati (Il bell'Antonio, 1949), e Lara Cardella (Volevo i pantaloni, 1989). Lo studioso mette in evidenza il legame che la mascolinità tradizionale intrattiene con la sottomissione della donna e con lo sguardo degli altri - fondamentale per convalidare o invalidare l'identità maschile. Tre i principali strumenti utilizzati in questo saggio: l'idea di performance (proposta da Judith Butler), l'idea di normalità (o invisibilità della mascolinità) e infine, la paura degli uomini di perdere la loro posizione dominante all'interno del patriarcato.
\end{abstract}

\section{RÉSUMÉ}

L'article analyse le lien qui s'établit entre le patriarcat et la masculinité dans les romans de Vitaliano Brancati (Il bell'Antonio, 1949) et Lara Cardella (Volevo i pantaloni, 1989). Le chercheur souligne le rapport entre la masculinité traditionnelle, la soumission de la femme et le regard des pairs - un regard déterminant pour valider ou invalider l'identité masculine. On dénombre trois outils principaux utilisés dans cet essais : l'idée de performance (proposée par Judith Butler), l’idée de normalité (ou invisibilité de la masculinité) et la crainte des hommes de perdre leur position dominante dans la société patriarcale.

I

n questo saggio non si vogliono analizzare i romanzi di Vitaliano Brancati (Il bell'Antonio, 1949) e Lara Cardella (Volevo i pantaloni, 1989) dal punto di vista letterario, ma quali rappresentazioni dell'evoluzione (o mancata tale) della mascolinità siciliana. Brancati sembra proporre un nuovo tipo di mascolinità attraverso l'ironia, usata per rendere palesi le imposizioni patriarcali sugli uomini siciliani. Questa nuova idea di mascolinità, come si vedrà in Lara Cardella che scrive quaranta anni dopo Brancati, non è ancora stata pienamente raggiunta. Alle soglie del Ventunesimo secolo, il patriarcato e l'idea di mascolinità messa alla berlina da Brancati, ancora esiste e resiste in Sicilia, e non sembra 
concedere spazio a nessuna alternativa di genere. In questo percorso di analisi saranno utilizzate le idee di performance (proposta da Judith Butler), l'idea di normalità (o invisibilità della mascolinità) e infine, la vera forza mortice del patriarcato: la paura degli uomini di perdere il loro potere ed essere ostracizzati dalla società patriarcale dominante. Parlando della perpetuazione dei ruoli di genere, Pierre Bourdieu ha scritto:

Le travail de reproduction était assuré [...] par trois instances principales, la famille, l'Église et l'École, qui, objectivement orchestrées, avaient en commun d'agir sur les structures inconscientes. C'est sans doute à la famille que revient le rôle principal dans la reproduction de la domination et de la vision masculines; c'est dans la famille que s'impose l'expérience précoce de la division sexuelle du travail et de la représentation légitime de cette division, garantie par le droit et inscrite dans le langage ${ }^{1}$.

Questa costruzione della "struttura" societaria (o "verità" nel senso foucaltiano del termine) ovviamente influenza i singoli membri, i loro comportamenti e i loro codici sociali. Per imporre questa struttura/verità bisogna che siano create delle "credenze" collettive. Nel caso del genere (gender) e della creazione di tali credenze molti studi hanno rivelato la differenza del linguaggio usato verso i bambini fin dalla nascita, un linguaggio che "genderizza" il bambino. Alessandro Taurino ha ribadito come "il linguaggio svolge pertanto importantissime funzioni, che mettono in campo componenti inerenti non solo alla sfera della identità dei soggetti ma, in modo particolare [...] alla dimensione dei rapporti tra gli individui nel contesto socio-relazionale" ${ }^{2}$.

Iniziando dal romanzo di Brancati, l'imposizione del patriarcato su Antonio appare evidente fin dalle prime pagine, quando Brancati racconta due episodi della vita del giovane protagonista. Il primo episodio fa intravedere anche una sottile omosessualità: una servetta è innamorata di Antonio e, ossessionata da questa passione, passa le notti dietro la sua porta. La madre chiede consiglio a Padre Giovanni che per Antonio nutriva "sentimenti non perfettamente cristiani"’3. L'ironia di Brancati, usata per mostrare i meccanismi patriarcali d'identità di genere e l'imposizione di tali schemi sui singoli individui, viene evidenziata ancor più chiaramente con il secondo episodio, quando Antonio, a

1. Bourdieu Pierre, La Domination masculine, Paris, Éditions du Seuil, 1998, p. 85.

2. TAurino Alessandro, Identità in transižione, Milano, Edizioni Unicopli, 2003, p. 149.

3. Brancati Vitaliano, Il bell'Antonio, Milano, Bompiani, 1949. Le citazioni sono tratte dall'edizione Milano, Mondadori Oscar Classici, 2001 (la presente, p. 9). 
Roma prima di ripartire per Catania per conoscere la futura moglie Barbara, incontra lo zio materno Ermenegildo Fasanaro. Lo zio senza esitazioni inquadra Antonio: "Sono sicuro che sei di quelli che darebbero un patrimonio pur di raggiungere ogni notte una cifra alta... È così o mi sbaglio?” La risposta di Antonio è solo: "Ma io in verità..." ed è la conferma della sua passiva assogettazione ai canoni del vecchio gallo, e per estensione, alle aspettative della società patriarcale siciliana. Lo zio è un alter ego del padre di Antonio, due uomini che sono ancora legati a un unico (e totalizzante) modello di mascolinità, che non ammette eccezioni. Come ha sostenuto Perrone, si tratta di una parte fondamentale della storia di Antonino che non è "come potrebbe a tutta prima apparire, la storia di un fiasco sessuale con la giovane moglie. Il suo insuccesso, ci vuol poco a capirlo, rimanda a una impossibilità più generale che fa crollare gli ultimi miti della generazione dei padri, di cui Alfio Magnano è irriducibile rappresentante" . Ad Antonio viene imposto il ruolo di Don Giovanni, e quindi è costretto a nascondere la sua anormalità di fronte alle aspettative della società. Il normale sembra cosi essere self-evident e perenne.

Una volta definita l'anormalità (ma ovviamente l'idea di anormalità è stata definita da chi ha il potere per definirla), la società siciliana "sana e normale" studia, categorizza gli anormali quasi ossessivamente, creando una relazione di potere assoluto del "normale" su "l'anormale". Le idee di Michel Foucault (di disciplina, normalizzazione e giudizio) possono tutte essere applicate a questo tipo di società siciliana. La forza della disciplina premia chi si attiene alle regole della società e rispetta il ruolo di brava figlia, o di brava moglie o di "gallo" nel nostro caso. Chi non si attiene a queste regole, allora, non è solo da riprovare ma è anormale (come in Brancati e Cardella) .

A questa idea si ricollega un'altra idea proposta da Judith Butler a proposito del gender quale performance (o esibizione). Nel Bell'Antonio e in Volevo i pantaloni, la costruzione del soggetto sessuale / sessuato avviene attraverso l'esibizione di tratti precostituiti che ne definiscono l'identità di genere. Si tratta di vere e proprie esibizioni, che non fanno altro che ribadire lo status quo della società, perpetuando le regole che premiano chi si adegua e ostracizzando chi si ribella,

\section{Ibid., p. 17.}

5. Perrone Domenica, Vitaliano Brancati. Le avventure morali e i "piaceri" della scrittura, Milano, Bompiani, 1997, p. 123.

6. Su questa dinamica, illuminante sembra il passaggio dal film documento Comiži d'amore (1965) di Pier Paolo Pasolini, quando intervista una donna di un cortile palermitano. Il regista chiede: "Le sembra giusta questa disparità tra uomini e donne?" La donna risponde: "È normale, lo fanno tutte e quindi non ce ne accorgiamo nemmeno". 
considerandolo anormale (quindi fuori dal discorso che mantiene la società normale) ed evitando anche di parlarne ${ }^{7}$.

Sono $\mathrm{i}$ linguaggi e i codici usati in queste opere che nel contesto di questo saggio sono interessanti; i semplici gesti e comportamenti dei vari personaggi, che attraverso la lente del genere, perdono quel loro carattere che Barthes ha definito "naturale" ma che invece sono il punto di partenza per smascherare e rivelare il meccanismo che trasforma la "culture petite bourgeoise en nature universelle" . Quella di Antonio è solo una performance che viene resa palese dopo il matrimonio con Barbara, un matrimonio non consumato. Antonio Magnano rappresenta quindi un caso emblematico di performance legata al genere, un uomo in cui la bellezza fisica viene appunto immediatamente identificata con virilità o potenza sessuale. Anche qui è fondamentale la performance, l'apparenza dell'essere gallo, l'essere forzati a una facciata di mascolinità. Dietro tale facciata si nasconde però la sua impotenza, una "colpa" imperdonabile nella società siciliana che assimila il fallo (sesso biologico) alla potenza sessuale9. È una società che definisce la mascolinità attraverso una serie di negazioni, come ha scritto Elisabeth Badinter nel suo XY De l'identité masculine, "la mascolinità è spesso definita attraverso il rifiuto di qualcosa piuttosto che attraverso il desiderio di qualcosa" ${ }^{\prime \prime}$. La colpa di Antonio è quella di cadere proprio nel classico dualismo negativo dei canoni patriarcali, e quindi nella paura dell'omofobia: il suo non essere attivo sessualmente con la moglie lo pone direttamente nella categoria negativa opposta, il non-essere-eterosessuale ovvero un non-maschio. Una definizione di genere al negativo, quindi: "Essere un uomo significa il non

7. Un altro film, che sembra ideale per spiegare questa idea di normalità del patriarcato e la sua accettazione da parte degli individui tramite il meccanismo della paura, è Divorżio allitaliana di Pietro Germi, 1961. Il protagonista Fefè (Marcello Mastroianni) usa proprio la paura dell'ostracismo (le armi del patriarcato per punire gli uomini che non obbediscono ai rigidi codici patriarcali) a suo vantaggio per poter utilizzare l'articolo 587 del Codice Penale (delitto d'onore) e avere una pena ridotta per l'omicidio di sua moglie.

8. Barthes Roland, Mythologies, Paris, Éditions du Seuil, 1957, p. 7.

9. Quelli che Alessandro Taurino chiama i "determinanti socio-culturali" a fondamento di una identità di genere che si contrappone alla ormai desueta "categoria del biologico, che riporta I fondamenti del sesso, de genere e sell'identità, al dato naturale, biologico, fisiologico, genetico"; TAURINO Alessandro, Identità in transiżione, cit., p. 17.

10. Badinter Elisabeth, X Y De l'identité masculine, Parigi, Odile Jacob, 1992, p. 173 (traduzione nostra). 
essere femminile, il non essere omosessuale; il non essere effeminato nella propria apparenza fisica o atteggiamento; il non essere impotente con le donne"11.

Il gallismo e la mascolinità siciliana, sono illusioni create attraverso il linguaggio che reinventa la realtà, arrivando a quella dislocazione dei personaggi di Brancati dal loro contesto patriarcale. Come ha scritto Spalanca, Brancati riduce il mito del Don Giovanni a pratica "essenzialmente immaginaria e verbale [...] una verbalità dalle gradazioni allusive, surreali, ed eroicomiche, è, infatti, il cardine di una fabula che accosta volutamente mediocrità e immaginazione, disadorna quotidianità e illusione" ${ }^{\prime 2}$. Per Simone de Beauvoir la donna "non esiste" e viene definita dall'uomo, nel nostro caso attraverso le infinite discussioni tra gli uomini siciliani nei romanzi di Brancati:

La donna si conosce e si sceglie non in quanto esiste di per sé ma in quanto è definita dall'uomo. Perciò è necessario anzitutto descriverla come gli uomini la sognano: perché il suo essere-per-gli-uomini è uno dei fattori essenziali della sua condizione concreta" ${ }^{13}$.

È questa lotta tra il vecchio modello e la nuova mascolinità proposta da Brancati, che sta alla base del romanzo. Nel personaggio di Antonio vengono rese palesi non solo le "regole" patriarcali, ma attraverso il loro smascheramento siamo all'inizio di un processo di ridefinizione dell'indentità di genere per il quale si dovrà aspettare il femminismo (vent'anni dopo il romanzo di Brancati) per costringere "gli uomini a mettere in atto meccanismi di ricollocazione della propria struttura identitaria fondati sul riconoscimento sia della fallacia di una presunta fissità di un modello maschile sia di un inevitabile declino dell'archetipo dell'eterno mascolino"'14. Dopo questi episodi il romanzo diventa più intimista, meno graffiante, segno appunto che Brancati sta elaborando una nuova idea di mascolinità in opposizione al patriarcato classico. Infatti, dopo la lunga introduzione di Antonio studente di legge a Roma, si ritorna a Catania, e per un attimo il gallo riemerge. A Catania, dove si parla delle prodezze sessuali di Antonio nella capitale, il padre ha già trovato per il figlio la futura sposa: Barbara Puglisi, figlia di un notaio catanese, colpisce Antonio che ne rimane quasi folgorato al primo

11. Ibid.

12. Perrone Domenica, Vitaliano Brancati: le avventure morali e i piaceri della scrittura, cit., p. 70.

13. De Beauvoir Simone, Il secondo sesso [1949], tr. it. Roberto Cantini e Mario Andreose, Milano, Il Saggiatore, 2002, p. 180.

14. Taurino Alessandro, Identità in transiz̨ione, cit., p. 118. 
sguardo. In questo passaggio, e nell'intero romanzo, sembra assente la classica ironia di Brancati. Qui lo scrittore mira invece a sottolineare il dramma di Antonio che non appartiene a quel modello patriarcale, e indaga "a fondo le motivazioni psicologiche del comportamento dei suoi conterranei, e probabilmente anche di se stesso" 15 . È la madre a dare il permesso di "ricambiare" lo sguardo del giovane Antonio:

La ragazza portava un libro da messa in mano e camminava un po' curva in avanti, stringendo a sé, e nascondendo nel modo più dolce, l'esuberanza e lo slancio della sua giovinezza. Una leggera gomitata della madre l'avvertì che poteva rimettere negli occhi, pudicamente diradati, lo sguardo e l'attenzione ${ }^{16}$.

La descrizione della reazione di Antonio somiglia a quella che si ha di fronte a una vera e propria apparizione e il paragone con le donne di Roma rappresenta una negazione assoluta per ogni altro tipo di donna che non si adegui all'immagine patriarcale imposta dalla società, in questo caso rappresentata dalla madre:

Un istantaneo vacillamento del passo la staccò dalla madre e la portò vicinissima al giovane che poté sentirne l'odore di velo, di pelle bruscamente riscaldata dal sangue, di forcine di tartaruga e d'indumenti conservati a lungo insieme a vecchi fiori, odore che nessuna donna di Roma aveva mai posseduto e che gli saettò dentro la carne come uno scotimento profondo ${ }^{17}$.

La nuova identità di genere sviluppata nel secondo romanzo di Brancati è infatti rappresentata non solo dal protagonista, Antonio, ma anche dal cugino, Edoardo Lentini, la cui delicatezza quasi femminile rappresenta l'unico conforto per il giovane ex-gallo catanese:

I due cugini rimasero tutta la sera al buio, vicino al balcone. Ogni tanto, per mettere un rumore qualunque nella stanza, Antonio tossicchiava, e dopo un poco, quasi a rispondere, Edoardo raschiava con la gola. In questo modo, passarono parecchie sere ${ }_{s}$ non avendo il coraggio di parlare a cuore aperto della terribile cosa che era accaduta ad uno di loro, non parlavano di nulla, ogni altro argomento trattato avrebbe fatto sentire maggiormente la gravità di quello che trascuravano ${ }^{18}$.

15. Abrugiati Luigia, Il primo tempo di Vitaliano Brancati, Lanciano, Carabba Editore, 1977, p. 83.

16. Brancati Vitaliano, Il bell'Antonio, cit., p. 42.

17. Ibid.

18. Ibid., p. 198. 
La netta divisione del romanzo tra prima e dopo lo scandalo dell'impotenza di Antonio, sembra rappresentare una divisione ideologica tra la vecchia mascolinità del gallo, e una nuova idea di maschio: Antonio, ancora innamorato di Barbara, stoicamente sopravvive alla più grave delle colpe nell'ambito patriarcale. Nella prima parte del romanzo Antonio è il dominatore:

Le donne si sentivano dominate e, insieme, a loro agio completo e perfetto: accanto a lui, bruciavano dolcissimamente, e soffrivano, e impazzivano con una soavità sì profonda da far pensare che una grave anomalia si fosse impadronita di esse confondendo il piacere e il dolore ${ }^{19}$.

Nella seconda parte del romanzo invece, Antonio viene giudicato e ostracizzato dalla società, ovviamente maschile, colpevole di non essere uno di loro, di essere diverso, anormale. Ma Brancati, per bocca del cugino Edoardo, presenta un'alternativa, una nuova possibilità di essere uomini in Sicilia:

Per qualunque persona di un altro Paese, sarebbe stato un incidente da nulla. Ma per noi no! per noi è una tragedia! perché noi pensiamo sempre a una cosa, a una sola cosa, a quella! e frattanto un tiranno ci caccia in guerra con una pedata nel sedere, e gli altri popoli ci ricacciano indietro con un'altra pedata, ed entrano nelle nostre case! Le donne, la donna!... Quattro volte, cinque volte, sei volte... Ecco gli oggetti delle nostre ansie!... Ma lo sai che non c'è nessun disonore a passare tutta la vita nella castità?... Sei bello, cortese, alto, forte, impari facilmente qualunque arte e scienza, sei in grado di capire tutto!... Ma pensa quante cose avresti potuto fare, se non ti fossi chiuso giorno e notte in un pensiero a consumarvi dentro la vita?

È nella domanda finale di Edoardo che si inquadra la nuova idea di mascolinità definita da Brancati. È la costrizione, la violenza subita dalla società e dai singoli per ottemperare alle regole sociali del patriarcato. Una domanda fondamentale perché segna il passaggio di Brancati dalla commedia del gallismo alla tragedia del gallismo quale devianza, malore patriarcale, espressa ed elaborata appieno nel terzo romanzo della trilogia, Paolo il caldo (1955). Alla fine del dialogo tra Antonio e Edoardo c'è un ultimo episodio che permette di leggere il patriarcato per quello che è, ovvero pura violenza sulle donne, ma anche della forza che esso mantiene su Antonio Magnano e sugli uomini siciliani. Siamo alla fine del romanzo e i due cugini si lasciano litigando perché Antonio non ha accettato la

19. Ibid., p. 6.

20. Ibid., p. 259. 
nuova identità di genere proposta dal cugino e vuole addirittura schiaffeggiare Barbara. Edoardo rincasa e la figlia del portiere, Giovanna, chiude la porta per paura dei soldati alleati. Edoardo la stupra:

Edoardo fu risoluto e sbrigativo, e non smise un solo istante la sua aria di uomo in collera. Quando si alzò e asciugò la fronte, chinò subito gli occhi, per non guardare la donna in faccia, vedendone già chiaramente, nei gesti con cui ella si stirava e batteva la gonna, la sorda stizza e la ribellione ${ }^{21}$.

Edoardo chiama al telefono il cugino Antonio per chiedere perdono e per raccontare l'accaduto e iniziando a piangere sente invece "sempre più intenso, scottante, attraverso quel filo del telefono [...] il calore di quell'invidia". Antonio inizia a piangere come un "adolescente tardivo". Non ci sono più parole tra $\mathrm{i}$ due, ed Edoardo "piano piano, delicatamente, chiuse il telefono"22. I due cugini sono quindi, alla fine del romanzo, degli ibridi, a metà strada di un cammino verso un'evoluzione della rigida mascolinità patriarcale che impone il dominio assoluto sulla donna, arrivando fino allo stupro. Judith Butler parla proprio di violenza nella creazione dell'identità eterosessuale, la stessa violenza che ritroviamo nella repressione dei possibili desideri omoerotici presenti nel secondo romanzo di Brancati. La violenza perpetua la creazione del maschio dominante, e al tempo stesso protegge la società e lo status quo ${ }^{23}$. Antonio e il cugino Edoardo esemplificano magistralmente come il processo per la creazione di una identità maschile si basi sulla repressione e sulla scelta ${ }^{24}$ : si privilegia una possibilità e se ne escludono altre. È una decisione personale? O è basata su una "norma" imposta dalla società che necessariamente deve categorizzare, normalizzare attraverso la dicotomia positivo - negativo, uomo - donna? Decostruire questo rigido pensiero dualistico sembra essere l'unica strada per affrontare il complesso problema dell'identità di genere, così come sembra suggerire Brancati. Nel Bell'Antonio la bellezza fisica di Antonio Magnano viene scambiata con virilità. Ma è solo apparenza, una performance per la società siciliana. Come visto in precedenza, dietro tale facciata si nasconde la "colpa" dell'impotenza, imperdonabile nella società siciliana. Un'idea questa che

21. Ibid., p. 262.

22. Ibid., p. 263.

23. ButLer Judith, Critica della violenza etica [2005], tr. it. Federico Rahola, Milano, Feltrinelli, 2006.

24. Cfr. Butler Judith, Bodies that Matter: on the Discursive Limits of "Sex", New York, Routledge, 1993. Vedi anche ButLer Judith, Gender Trouble, New York-London, Routledge, 1990. 
troviamo ripetuta anche negli anni ottanta nel romanzo di Lara Cardella. Come ha scritto Roland Barthes nel suo Mythologies (1957) quello che sembra del tutto naturale in una cultura (l'atteggiamento dongiovannesco in questo caso) è interamente costruito da contingenze storiche e sociali. Il maschio siciliano crea la propria identità di genere attraverso una performance che si adegua scrupolosamente ai dettami della società su come debba comportarsi un uomo: sono gli atti che ripetuti seguendo le convenzioni sociali creano il genere del singolo individuo (come nel personaggio di Piero) ${ }^{25}$.

Nella normalità della società patriarcale, la donna rimane assente, o meglio relegata al suo ruolo sussidiario di madre o moglie, e anche loro, nei romanzi di Brancati e Cardella, difendono lo status quo del patriarcato, che sembra, appunto, normale e inevitabile:

The absence of a formal institutional structure and of authorities, invested with the power to carry out institutional directives, creates the impression that the production of femininity is either entirely voluntary or natural $[\ldots]$ This system aims at turning women into the docile and compliant companions of men jutst as surely ad the army aims to turn its raw recruits into soldiers ${ }^{26}$.

È quello che accade in Volevo $i$ pantaloni. Lara Cardella fa da contraltare a Brancati per il suo stile crudo, diretto, dove in pieni anni Ottanta si vede ancora una Sicilia patriarcale e arcaica che "glorifica il fallo" 27 alla base della distinzione tra i sessi. Attraverso i suoi personaggi di un realismo sconcertante, l'immagine patriarcale della mascolinità siciliana si presenta ai nostri occhi alle porte del ventunesimo secolo, ancora come una realtà foucaltianamente naturale, o ancora, come ha scritto Stephen Goldberg "patriarchal societies were natural, biologically ordained and, thus inevitable"28. I concetti di naturalità e inevitabilità sono enunciati in un passaggio in particolare: "Ma com'era $\mathrm{o}$, meglio, chi era un uomo? Sentivo spesso ripetere, da mio padre, mia madre, i miei zii, frasi

25. Cardella Lara, Fedra se ne va, Milano, Mondadori, 1992.

26. Lee Bartky Sandra, "Foucault, Femininity and the Modernization of Patriarchal Power", in Conboy Katie, Medina Nadia, Stanbury Sarah (a cura di), Writing on the Body: Female Embodiment and Feminist Theory, New York, Columbia University Press, 1997, p. 143.

27. Gilmore David, "Introduction: the Shame of Dishonor", in Honor and Shame and the Unity of the Mediterranean, GiLmore David (a cura di), Washington, D.C., American Anthropological Association, 1987, p. 3.

28. Goldberg Stephen, The Inevitability of Patriarchy, New York, William Morrow and Company, 1973, p. 3. 
quali: 'un chiagniri... Tu si un masculu...' oppure 'Un masculu un ioca 'cchi fimmini' o ancora 'Talè a varvuzza ci sta spuntannu" 29 .

La repressione e la violenza nella società siciliana si manifestano poi anche attraverso il cosiddetto occhio sociale. Nelle opere di Brancati e Cardella viene descritta una società patriarcale dove la comunità stessa funge da garante (e esecutrice) dello status quo attraverso il controllo esercitato dall'occhio sociale. I ruoli e i comportamenti sono fissati rigidamente secondo un ordine binario (uomo, donna), e le eccezioni, gli ibridi, non sono ammessi. Parafrasando Foucault e la sua idea di Panopticon, nella società siciliana tutti guardano tutti, e l'intero nucleo comunitario finisce con l'assumere il ruolo di controllore ma automaticamente anche quello di soggetto controllato. Così facendo si giunge in pratica a un Panopticon Totale ${ }^{30}$. Lo sguardo e l'atto dell'osservare, oltre ai significati connotativi che a questi ultimi sono legati, sono presenti in maniera rilevante nei due testi letterari qui presi in analisi. La donna all'interno della famiglia descritta da Lara Cardella, diventa perciò un oggetto mentre allo stesso tempo finisce con l'essere da se stessa oggettivata, ricoprendo il doppio ruolo di vittima e carnefice delle leggi patriarcali: "Una delle funzioni più importanti dell'ideologia è quella di coprire le chiare relazioni di potere all'interno di una specifica società in un particolare momento storico, facendo apparire tali relazioni quale parte di un ordine naturale ed eterno" 31 .

Lo sguardo è un elemento fondamentale per la cultura meridionale e, anche in questo caso, il riferimento è duplice: sia allo sguardo negativo (il malocchio) $\mathrm{ma}$ anche allo sguardo costante della società sull'individuo. Foucault, in Sorveglire $e$ punire, si riferisce appunto al cosiddetto power of gaze, cioè il potere dello sguardo: "C'est le fait d'être vu sans cesse, de pouvoir toujours être vu, qui maintient dans son assujettissement lindividu disciplinaire" 32 . Il guardare, l'osservare e l'essere osservati, così importante e onnipresente nella società siciliana, insieme al linguaggio (verbale e corporale) creano questo stato di perenne analisi, da un lato verso l'esterno (quando si osserva), dall'altro verso l'interno (quando si è osservati). Questo discorso è valido soprattutto nelle piccole

29. Cardella Lara, Volevo i pantaloni, Mondadori, Milano, 1994, p. 21.

30. Ventura Renato, Mascolinità all'italiana: cinema teatro letteratura, Cuneo, Nerosubianco, 2013.

31. Nochlin Linda, "Women Art and Power", in Feagin Susan, Maynard Patrick (a cura di), Aesthetics, Oxford-New York, Oxford University Press, 1997, p. 72. Traduzione nostra.

32. Foucault Michel, Surveiller et punir. Naissance de la prison, Paris, Gallimard, 1975, p. 189. 
comunità (descritte da Brancati e Cardella), dove l'individuo è creato dalle abitudini sociali, ovvero dalla tradizione dei gesti ripetuti. Più stretta è la conoscenza reciproca, più forte è lo sguardo della società, più difficile è uscire fuori dai suoi canoni e quindi ogni violazione dello stretto codice si risolve spesso in violenza, non solo fisica ma anche verbale. Esemplare, a questo proposito, l'episodio descritto in Volevo i pantaloni, quando la giovane Lara viene redarguita a suon di ceffoni dal preside della scuola perché truccata. Le madri diventano esse stesse le rigide esecutrici del patriarcato: "Le madri chiudevano in casa le figlie e quando andavano a far la spesa tenevano gli occhi bassi per la vergogna, per il disonore" 33 . I padri invece, rincarano la dose con gli insulti, e l'insulto da parte di un singolo rappresenta la voce di una intera comunità che "sottomette" la donna, con buttana. Parlando del termine Queer Judith Butler ha scritto che la forza dell'insulto deriva da

[...] repeated invocation by which is has become linked to accusation, pathologization, insult. This is an invocation by which a social bond among homophobic communities is formed through time. The interpellation echoes past interpellations, and binds the speakers, as if they spoke in unison across time. In this sense it is always an imaginary chorus that taunts "Queer"

In conclusione, parafrasando Althusser a proposito di ideologia e normalità, la realtà della società siciliana descritta nei romanzi di Brancati e Cardella è illusione che allude a una idea di mascolinità che interpella gli individui (uomini e donne insieme), e li sprona ad agire come soggetti sottomessi volontariamente alle regole societarie non scritte:

Mais l'immense majorité des (bons) sujets marchent bien "tous seuls", c'està-dire à l'idéologie (dont les formes concrètes sont réalisées dans les Appareils idéologiques d'État). Ils s’insèrent dans les pratiques, gouvernées par les rituels des AIE. [...] l'individu est interpellé en sujet (libre) pour qu'il se soumette librement aux ordres du Sujet, donc pour qu'il accepte (librement) son assujettissement, donc pour qu'il "accomplisse tout seul" les gests et actes de son assujettissement ${ }^{35}$.

33. Cardella Lara, Volevo i pantaloni, cit., p. 11.

34. ButLer Judith, Bodies that Matter: on the Discursive Limits of "Sex", cit., p. 226.

35. Althusser Louis, Sur la reproduction, Paris, PUF, 1995, p. 311. Corsivo nel testo. 
Una pratica che ancora nel 1989, come abbiamo visto nel romanzo di Lara Cardella, esiste e resiste in Sicilia nonostante la lotta femminista e l'affermarsi di modelli alternativi di mascolinità.

Renato VentuRA

University of Dayton 